\title{
Pais por Inteiro: Avaliação Preliminar da Eficácia de Uma Intervenção em Grupo para Pais Divorciados
}

\author{
Preliminary Efficacy Evaluation of a Group Intervention Program \\ for Divorced Parents
}

\author{
Diogo Lamela ${ }^{*}, a, b$, Maria Castro $^{a}, \&$ Bárbara Figueiredo $^{a}$ \\ ${ }^{a}$ Universidade do Minho \\ ${ }^{b}$ Instituto Politécnico de Viana do Castelo
}

\begin{abstract}
Resumo
O artigo trata da avaliação preliminar da eficácia do Pais por Inteiro (PApi), uma intervenção em grupo com pais divorciados, implementada com a finalidade de melhorar a adaptação ao divórcio, a coparentalidade e a binuclearidade familiar. Os participantes $(n=16)$ foram equitativamente distribuídos em um grupo experimental (sujeito a intervenção) e um grupo de controlo. Os grupos mostraram-se semelhantes nas medidas dependentes pré-intervenção. Os resultados evidenciaram uma melhoria entre o pré e pós-teste no ajustamento ao divórcio e na qualidade da coparentalidade no grupo experimental, e uma melhoria no pós-teste no ajustamento também no grupo experimental. Estas melhorias não existiram no grupo de controlo. Estes resultados sugerem a eficácia do PApi em termos do melhor ajustamento pósdivórcio e coparentalidade.

Palavras-chave: Intervenção em grupo; Divórcio; Adaptação; Coparentalidade; Binuclearidade.

Abstract

The article deals with the preliminary evaluation of the efficacy of a group intervention program composed by divorced parents, named Pais Por Inteiro (PApi), which was implemented with the aim of improving adjustment to divorce, coparenting relationship and family binuclearity. Participants $(n=16)$ were equally distributed in an experimental group (subject to intervention) and a control group. Groups were similar in pre-intervention dependent measures. The results showed an improvement between preand post-test in adjustment to divorce and coparental quality in the experimental group, and an improvement in post-test in adjustment in the experimental group. Those improvements did not exist in the control group. The results suggest the efficacy of PApi in terms of better post-divorce adjustment and coparental relations.

Keywords: Group intervention; Divorce; Adjustment; Coparenting; Binuclearlity.
\end{abstract}

O divórcio é uma transição familiar cada vez mais comum nas sociedades ocidentais. Seguindo esta tendência, a taxa de divórcio em Portugal tem aumentado gradualmente nas últimas décadas. Os dados mais recentes cifram a taxa de divorcialidade portuguesa em $2,4 \%$, o que significa, considerando o número de casamentos em 2006, que há cerca de 4,9 divórcios por 10 casamentos, ou seja, quase metade dos casamentos terminam em divórcio (Instituto Nacional de Estatística [INE], 2008). Trata-se de um aumento relevante, uma vez que em 2000 apenas se registavam 3 divórcios por 10 casamentos celebrados.

\footnotetext{
* Endereço para correspondência: Universidade do Minho, Escola de Psicologia, Serviço de Consulta Psicológica e Desenvolvimento Humano - Unidade de Crianças e Adolescentes, Campus Gualtar, Braga, Portugal, 4710-057.E-mail: dlamela@ese.ipvc.pt
}

Os dados evidenciam que os primeiros 10 anos são críticos para a manutenção da relação conjugal, uma vez que 4 em cada 10 casais que se divorciam o fazem na primeira década de casamento (INE, 2001, 2008) Mais especificamente, $18 \%$ dos divórcios ocorrem nos primeiros quatro anos de vida conjugal.

Embora seja um fenómeno frequente nas sociedades ocidentais, o divórcio caracteriza-se por ser um momento de elevada exigência, em termos de adaptação social e psicológica dos diferentes elementos da família (Hetherington \& Kelly, 2002; Lamela, 2009; Wang \& Amato, 2000). A adaptação ao divórcio é o resultado da interacção de múltiplos mediadores psicossociais, condicionada por características individuais e pelo contexto desenvolvimental.

Os recém-divorciados estão sobretudo centrados no ajustamento instrumental à dissolução conjugal; medidas de ordem prática são tomadas, como, por exemplo, a 
reorganização financeira, a mudança de casa de um dos elementos do ex-casal, a legalização do divórcio, a regulação do poder parental, os cuidados aos filhos (Sweeper, 2004; Wang et al., 2000). Neste primeiro momento, a principal finalidade dos pais é proteger e atenuar os efeitos do divórcio nos filhos. Todavia, esta é uma tarefa muito difícil - e, na maioria dos casos, mal sucedida. Evidências empíricas comprovam que grande parte dos pais não consegue separar a relação conjugal do passado da relação coparental do presente, nos primeiros dois anos de divórcio (Ferrante, 2005; Maccoby, Depner, \& Mnookin, 1990). Algumas vezes, observa-se uma coparentalidade paralela, em que, embora ambos os pais estejam envolvidos nos cuidados prestados aos filhos, têm uma prática parental descoordenada entre si, caracterizada pela inexistência de comunicação, o que leva a criança a viver em dois mundos sociais separados, sem consistência de regras, reforços, valores e oportunidades desenvolvimentais.

A compreensão dos factores que contribuem para a adaptação ao divórcio não é linear. O estudo longitudinal de Hetherington (2003), por exemplo, identifica dois grandes rumos desenvolvimentais. No primeiro, considerado o mais adaptativo, o divórcio é conceptualizado como um momento agudo de stress, similar a uma crise moratória, em que, após os dois primeiros anos (em média), os níveis de ajustamento e bem-estar psicológico são iguais ou superiores aos níveis anteriores à disrupção conjugal. No entanto, num segundo possível trajecto desenvolvimental, o divórcio poderá significar um stressor crónico, com repercussões ao longo da vida, em que não é evidente uma evolução adaptativa a esta transição familiar.

Dado este período poder representar um momento de desestabilização emocional e risco psicopatológico, foram propostos programas de intervenção, com a finalidade de auxiliar as famílias a enfrentar adaptativamente os desafios desenvolvimentais trazidos pela dissolução conjugal. Tais programas visam, por um lado, a redução de padrões comunicacionais e relacionais disfuncionais e destrutivos e, por outro lado, a promoção da criação de novos limites relacionais, novas fronteiras e reorganização dos vínculos emocionais (Blaisure \& Geasler, 2000; Cookston, Braver, Griffins, Lusé, \& Miles, 2007; Geasler \& Blaisure, 1998; Sbarra \& Emery, 2006).

De uma forma global, a investigação tem evidenciado a eficácia das intervenções com uma matriz educacional e psicoterapêutica, quer no equilíbrio e bem-estar psicológico dos pais, na diminuição dos factores e padrões de risco, no fortalecimento dos factores protectores através do empowerment ${ }^{l}$ no treinamento de estratégias de reso-

${ }^{1} \mathrm{O}$ empowerment é conceptualmente definido como o processo de aumento do sentido de competência das pessoas para tomarem decisões e transformar essas decisões em acções e comportamentos de participação que lhes aumentem o bem-estar, a qualidade de vida e sentimentos de eficácia e controlo (Zimmerman, 1995). lução de problemas, na dinamização e mobilização dos recursos da rede social, na atenuação e diluição do conflito interparental e na transformação da coparentalidade paralela em coparentalidade cooperante, positiva e ajustada às necessidades desenvolvimentais dos filhos (Blaisure \& Geasler, 2006).

Em 2004, Gilmour reviu as características mais salientes e eficazes dos programas de intervenção com pais e concluiu que a maior parte assume um carácter psicoeducacional, apostando na aprendizagem de competências parentais e de técnicas comportamentais de gestão e controlo do conflito. A autora concluiu que a maioria dos programas foca-se apenas em proporcionar aos pais conhecimentos sobre o processo legal do divórcio, as opções de litígio e informação psicológica sobre o impacto negativo que divórcios mais conflituosos, e, por consequência, mais disruptivos, podem ter no desenvolvimento da criança (Gilmour, 2004). Dessa forma, argumenta que a grande parte dos programas analisados, que se caracterizam como psicoeducacionais, na realidade devem ser considerados como meramente informacionais, uma vez que não têm uma intenção directa de promover mudanças psicoterapêuticas nos participantes. Nesta linha orientadora, Stewart (2001) recomenda preocupação na interpretação das melhorias alcançadas pelos participantes.

Avaliando alguns dos estudos publicados que apresentaram resultados psicoterapêuticos positivos, poder-se-á inferir três denominadores comuns dessas intervenções (Ahrons, 1981; Arbuthnot, Poole, \& Gordon, 1996; Bloom, Hodges, \& Caldwell, 1982; Kramer, Arbuthnot, Gordon, Rousis, \& Hoza, 1998; Pereira \& Matos, 2008; Stone, McKenry, \& Clark, 1999). A primeira característica dos programas com eficácia terapêutica prende-se com a formação de um espaço de partilha experiencial, para os participantes terem oportunidade de reflectir e discutir, num ambiente de confiança, os sentimentos e emoções em relação à disrupção conjugal e ao período de adaptação consequente (Geasler et al., 1998; Øygard, 2004; Øygard, Thuen, \& Solvang, 2000). O segundo denominador comum é a utilização de métodos de intervenção centrados na promoção das capacidades e competências dos participantes (Blaisure et al., 2006; Gilmour, 2004). O terceiro e último factor comum consiste no provimento de informação psicológica sobre as etapas, processos, causas e consequências do divórcio (Frieman, Garon, \& Garon, 2000).

Nesta sequência, Øygard et al. (2000) averiguaram que os grupos de intervenção, independentemente das suas orientações teóricas e objectivos terapêuticos, deveriam proporcionar quatro elementos essenciais: suporte e compreensão experiencial, partilha experiencial, um sentimento de pertença e coesão e, finalmente, um sentimento de protecção e de segurança. Na opinião destes autores, a dimensão experiencial é o pilar basilar da mudança terapêutica. 
Numa outra revisão bibliográfica sobre programas norte-americanos destinados a pais divorciados, Geasler e Blaisure (1998) concluíram que é essencial, neste tipo de intervenção em grupo, o treinamento de capacidades e competências na promoção de um comportamento coparental mais efectivo. Estas autoras utilizam como exemplo o estudo de Arbuthnot et al. (1996), que demonstra que programas centrados na promoção de competências promovem mais eficazmente a mudança do comportamento coparental, em comparação com programas que utilizam estratégias de intervenção mais passivas como, por exemplo, seminários e workshops ou brochuras. Por consequência, os programas interventivos centrados nas competências permitem aos pais aumentar a sua capacidade de escolher formas de comunicação e interacção mais positivas (Gilmour, 2004; Goodman, Bonds, Sandler, \& Braver, 2004) e os seus efeitos são mais duradouros em comparação aos programas educacionais tradicionais (Blaisure et al., 2006).

Os programas de intervenção em grupo com pais divorciados podem incidir sobre dois focos distintos: programas de intervenção centrados nos filhos e programas interventivos centrados nos pais. A intervenção centrada nos filhos tem como finalidade auxiliar os pais na melhoria do ajustamento geral dos filhos ao divórcio. Estes programas não se preocupam directamente com a adaptação dos pais e não promovem o seu desenvolvimento enquanto indivíduos. Por sua vez, os programas de intervenção em grupo centrados nos pais têm, como finalidade psicoterapêutica, intervir em todos os papéis que os participantes desempenham. Por outras palavras, utilizam conteúdos e métodos de intervenção que proporcionam aos participantes desenvolverem-se como pais, mas também como pessoas que operam num contexto sociocultural (Gilmour, 2004).

A partir do exposto, este estudo tem como objectivo geral realizar uma avaliação preliminar da eficácia da primeira versão do Pais por Inteiro, um programa desenvolvimental de intervenção em grupo destinado a pais divorciados. O PApi, pelas características que irão ser descritas seguidamente, apresenta uma matriz desenvolvimental por, em primeiro, conceptualizar o divórcio como uma transição desenvolvimental que potencia um conjunto de transformações e mudanças quantitativas e qualitativas nas trajectórias dos adultos, em segundo, orientar os objectivos, métodos e finalidades da intervenção considerando as tarefas desenvolvimentais da idade adulta (Arnett, 2001; Lachman, 2001) e, em terceiro lugar, seguindo os construtos teóricos da Ciência Desenvolvimental Aplicada (Lerner, 2006), identificar, promover e desenvolver um conjunto de activos interpessoais e ambientais que potenciem resultados desenvolvimentais positivos nos pais e, indirectamente, nas crianças e nas famílias.

\section{Método}

\section{Recrutamento e Selecção dos Participantes}

O recrutamento dos participantes foi feito através de dois procedimentos distintos. Foram seleccionados todos os pais divorciados com filhos que foram acompanhados no Serviço de Consulta Psicológica e Desenvolvimento Humano da Escola de Psicologia da Universidade do Minho (SCPDH-UM) ${ }^{2}$, e cujo processo terapêutico estava finalizado. Foi também lançada uma campanha de publicitação do programa em Serviços de Saúde públicos e privados na cidade de Braga, Portugal. Os pais interessados em receber mais informações deveriam telefonar para a recepção deste serviço universitário de consulta psicológica, fazer uma pré-inscrição e aguardar pelo contacto de um dos responsáveis pelo programa. Deste protocolo de recrutamento, foram identificados 22 pais divorciados, sendo que 18 resultaram do levantamento dos casos em consulta neste serviço e 4 de pré-inscrições por telefone, resultante da publicitação levada a cabo noutras instituições.

Uma entrevista de selecção telefónica estandardizada teve lugar com a finalidade de recolher algumas informações sócio-demográficas (e.g. idade, escolaridade, local de residência), assim como avaliar a presença dos seguintes requisitos de exclusão: (a) divórcio ocorrido num período inferior a um ano e meio ou superior a 12 anos; (b) filhos com idade superior a 18 anos; (c) processo actual de acompanhamento psicológico quer dos pais, quer dos filhos; (d) recasamento ou união de facto; (e) filho com diagnóstico de deficiência mental ou perturbações globais do desenvolvimento; (f) os pais residirem a mais de uma hora do local da intervenção; $(\mathrm{g})$ os pais não terem disponibilidade no horário estipulado para a ocorrência da intervenção.

Devido ao limitado número de pais que preenchiam todos os critérios de inclusão para participar no estudo, não foi possível dividir a amostra aleatoriamente em grupo experimental e grupo controlo. $\mathrm{O}$ grupo de controlo foi seleccionado por métodos não-probabilísticos, através de uma selecção por conveniência e era composto por pais que, devido ao horário agendado das sessões, não tinham disponibilidade para participar; no entanto, consentiram participar no estudo, preenchendo os instrumentos de auto-relato. Foram escolhidos sujeitos com características sócio-demográficas semelhantes às dos participantes do grupo experimental (idade, sexo e número de anos em que o ocorreu o divórcio), uma vez que 
estas variáveis, caso não controladas, poderiam por si só explicar as diferenças entre os grupos após a aplicação do programa.

\section{Amostra}

O grupo experimental foi constituído por 8 participantes, 5 do sexo feminino e 3 do sexo masculino. As idades variaram entre os 32 e os 40 anos, sendo que a média das idades era de 35,13 anos (desvio-padrão 2,95). O divórcio ocorreu, em média, há 4 anos. Quatro dos participantes estudaram até ao $12^{\circ}$ ano inclusive (50\%), 3 são licenciados $(37,5 \%)$ e 1 tem uma pós-graduação $(12,5 \%)$. No momento de avaliação, 5 tinham um agregado familiar composto pelo próprio e os filhos $(62,5 \%)$, sendo que 6 mantinham uma relação íntima (75\%). Na regulação do poder paternal, 7 dos participantes pertenciam a um regime de guarda única $(87,5 \%)$, enquanto, para um dos participantes, o regime era de guarda partilhada $(12,5 \%)$. A idade dos filhos dos participantes variava entre os 3 meses de idade e os 17 anos, sendo que a média situava-se nos 8,44 anos (desvio-padrão 3,98).

O grupo de controlo era também constituído por 8 participantes, 4 do sexo masculino e 4 do sexo feminino, com idades a variar entre os 28 e os 49 anos, apresentando uma média de 38,88 anos (desvio-padrão 6,98). Em média, o divórcio ocorreu a 6.5 anos. Cinco tinham uma frequência académica menor ou igual ao $12^{\circ}$ ano $(62,5 \%)$ e 3 eram licenciados $(37,5 \%)$. Seis dos participantes (75\%) tinham um agregado familiar composto pelo próprio e pelos filhos. Neste grupo, 4 dos 8 participantes mantinham uma relação íntima no presente. Todos os sujeitos do grupo do controlo regiam-se pela regulação do poder paternal de guarda única. A idade dos filhos dos participantes situava-se entre os 4 e os 17 anos, sendo que a média era de 11.42 (desvio-padrão 3,79).

Em ambos os grupos apenas participaram um dos excônjuges, não sendo possível a participação de uma díade parental (pai e mãe divorciados) quer no grupo experimental, quer no grupo de controlo.

\section{Procedimento}

Os participantes, após assinarem um consentimento informado que explicava os principais objectivos e procedimentos da investigação e do programa de intervenção, completaram em dois momentos os questionários que constituíram a bateria de avaliação da eficácia do programa: no pré-teste (momento 1, na semana da primeira sessão semanal do programa) e pós-teste (momento 2, na semana da ultima sessão do programa). O grupo de controlo completou a bateria de avaliacao nos mesmos momentos temporais que o grupo experimental. Neste caso, os questionários foram enviados para a casa dos pais por correio postal com um envelope pré-pago para o retorno dos questionários.

A todos os participantes foi pedido que respondessem aos questionários o mais sinceramente possível e que considerassem com atenção as informações prestadas. No momento 1 de avaliação (pré-teste), no grupo experimental, um dinamizador do grupo leu as instruções e assistiu os pais que tinham alguma dificuldade em compreender as questões. No grupo do controlo, foram enviadas por escrito as mesmas informações prestadas oralmente ao grupo experimental. Em ambas as situações, os pais foram informados que as suas respostas eram confidenciais.

A bateria de avaliação da eficácia do programa era constituída por um questionário de levantamento sociodemográfico, por um instrumento de avaliação da psicopatologia geral e do bem-estar psicológico (Brief Symptom Inventory), uma escala para medir a aliança coparental (Parenting Alliance Measure) e um inventário para avaliar a adaptação ao divórcio (Divorce Adjustment Inventory Revised) que foram administrados por esta ordem.

O Pais por Inteiro (PApi) foi desenvolvido durante 8 sessões semanais, com duração de aproximadamente de 90 minutos, sempre à mesma hora e dia da semana numa sala de intervenção em grupo do Serviço de Consulta Psicológica e Desenvolvimento Humano da Universidade do Minho. Os objectivos, conteúdos e actividades de cada sessão estavam claramente planificados no PApi - Manual de Aplicação (Lamela, Gonçalves, Castro, \& Figueiredo, 2007b) e serão descritos brevemente de seguida.

\section{Delineamento da Intervenção e Materiais}

Pais por Inteiro é uma intervenção breve a ser conduzida em grupo com pais divorciados. O programa, descrito por Lamela et al. (2007b), tem como finalidade não só auxiliar os pais na melhoria da sua relação de coparentalidade com o ex-cônjuge, como também contribuir para a sua adaptação mais ajustada a este momento de transição desenvolvimental (Lamela, Gonçalves, Castro, \& Figueiredo, 2007c). Este trabalho é feito através da discussão, partilha experiencial, aprendizagem de técnicas de gestão e evitamento de conflito, técnicas de planificação de um plano parental, acompanhadas por momentos de role-play para treino efectivo das competências desenvolvidas. O objectivo é impulsionar os factores protectores que medeiam a adaptação ao divórcio, fortalecer trajectórias pessoais satisfatórias e diluir processos de conflito interparental que diminuam a criação de aliança parental pós-dissolução conjugal (Lamela et al., 2007c). Os objectivos e estratégias de intervenção encontram-se sintetizados na Tabela 1 .

De acordo com a tipologia dos níveis de intervenção em grupo com pais divorciados proposta por Blaisure e Geasler (2006), o PApi preenche todos os critérios para ser considerado como um programa com um nível intenso de intervenção psicoeducacional e psicoterapêutica, uma vez que, devido aos seus objectivos, estrutura, organização, currículo e recursos despendidos, proporciona 
Tabela 1

Objectivos e Estratégias de Intervenção do PApi (versão 1)

\section{Objectivos}

Técnicas/Estratégias

$\begin{array}{ll}\text { Sessão } 1 & - \text { Construir um sentimento de coesão grupal } \\ \text { Apresentação } & \text { entre os participantes. } \\ \text { e Representação } & - \text { Elaborar as regras de funcionamento do grupo. } \\ & - \text { Debater o papel esperado dos dinamizadores } \\ & \text { e dos participantes. }\end{array}$

Sessão 2

Um acordo para

o cuidado:

Missão (Im)Possível?

(Parte I - Os

comportamentos)
- Reconhecer as pistas comportamentais de conflito interpessoal.

- Levar os pais a reflectirem sobre os mecanismos de conflito que estão presentes na sua vida.

- Compreender o impacto do conflito parental exerce no comportamento e nas emoções dos filhos e dos próprios pais.
- Actividades de apresentação e de promoção do sentimento de grupo.

- Apresentação e debate sobre os conteúdos propostos pelo PApi.

- Q-Set sobre técnicas passivas de resolução de conflito.

- Discussão sobre o conceito de conflito, causas dos conflitos e o impacto do conflito na família

- Treino em role-play de identificação das pistas que indiciam um conflito.
Sessão 3

Um acordo para

o cuidado:

Missão (Im)Possível?

(Parte II - As emoções)
- Compreender que o divórcio não é um processo apenas legal, mas também emocional.

- Debater os sentimentos, expectativas sobre o divórcio de cada participante para desenvolver um espaço de partilha e aceitação experienciais
- Partilha aberta das dúvidas, sentimentos, opiniões e vivências de cada participante quanto ao divórcio.

- Actividade: Será mesmo que estoudivorciado/a do meu casamento?

\section{Sessão 4}

Adeus até sempre:

Um mapa precisa-se!

Sessão 5

Gerindo emoções...
- Reflectir sobre como os sentimentos negativos (como o ressentimento e raiva) ainda os vincula ao casamento. Reflectir sobre a adaptabilidade destes sentimentos.

- Perceber até que ponto as disputas conjugais podem distorcer a imagem parental do ex-cônjuge.
- A Árvore do futuro: os participantes racionalizam as perdas e os ganhos do divórcio.

- Actividade: "Eu daqui a 5 anos, como quero ser? O que é que preciso para atingi-lo?"

- Actividade: "Daqui a 5 anos, que tipo de relação terei com o meu ex-cônjuge?"

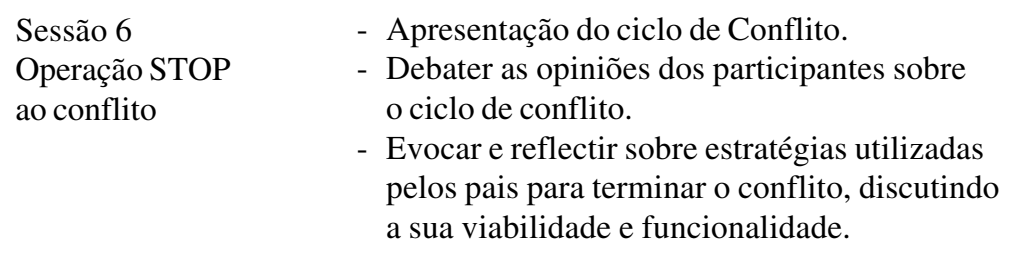

Sessão 7

Palavra-chave:

NEGOCIAR

e negociar, a bem

dos nossos filhos
- Levar a perceber os benefícios de uma negociação coparental para os filhos.

- Reflectir sobre o processo de (não) negociação de cada participante e retirar exemplos para o grupo do que é uma boa negociação coparental e o que é uma negativa negociação coparental.

- Apresentar técnicas efectivas de negociação diária entre os dois pais.
- Aprendizagem de técnicas de gestão e controlo do conflito interparental.

- Treino em role-play das técnicas de gestão e conflito interparental.
- Apresentação do conceito de Plano Parental.

- Apoio na elaboração de um Plano Parental seguindo a regra dos 6 factores.

\section{Sessão 8}

Sermos pais em conjunto: Uma equação para toda a vida
- Debater as consequências que a participação do PApi terá na vida dos participantes.

- Fortalecer a nova rede de contacto criada entre os participantes do programa.
- Delineamento de objectivos a curto e longo prazo de relação com o ex-cônjuge: "Quais são os passos que me levarão a alcançá-lo?"

aos participantes um envolvimento parental intenso, com possibilidade de encontrarem métodos e estratégias de coping em situações de potencial tensão emocional, fa- cultando espaço para treinar competências essenciais na atenuação ou diluição dos conflitos parentais. Além disso, uma vez que as sessões se desenrolam em pequenos 
grupos, potencializa e estimula a partilha experiencial facilitada por terapeutas com competências de aconselhamento clínico em grupo.

Conforme já mencionado, o programa consistiu em 8 sessões semanais de 90 minutos. Providenciou-se um espaço onde os pais poderiam deixar os filhos enquanto participavam das sessões do PApi. Este espaço não foi utilizado para qualquer tipo de intervenção terapêutica dirigida às crianças. Os terapeutas do grupo utilizaram o PApi-Manual de Aplicação (Lamela et al., 2007b) como guia para o desenvolvimento das sessões. Este manual descreve os objectivos e estratégias para cada sessão, os resultados desejados e os exercícios específicos que devem ser usados para atingir esses resultados. Os participantes no final das sessões receberam pequenas fichas informacionais relacionadas com o tema tratado na sessão em questão. Estas fichas permitiam que os pais se relembrassem e aplicassem os conhecimentos e técnicas discutidas no grupo às suas situações diárias.

\section{Instrumentos}

Questionário de Levantamento Socio-Demográfico ([QLSD], Lamela, Gonçalves, Castro, \& Figueiredo, 2007a). Este questionário de 18 itens tinha como finalidade recolher informação sobre os domínios da vida dos participantes relevantes para o estudo, como, por exemplo, informações sobre o divórcio e arranjos legais acordados e características sócio-demográficas dos participantes e dos filhos.

Brief Symptom Inventory ([BSI], Derogatis \& Melisaratos, 1983; versão portuguesa de Canavarro, 1999). O BSI, uma versão reduzida do Symptom Checklist90-Revised, é uma medida de avaliação do distress e mal-estar psicológico em adultos, através da quantificação dos sintomas de psicopatologia. Com 53 itens, avalia 9 dimensões psicopatológicas (somatização, obsessão-compulsão, sensibilidade interpessoal, depressão, ansiedade, hostilidade, ansiedade fóbica, ideação paranóide e psicoticismo). As características psicométricas da versão portuguesa apresentam valores de consistência interna bastante satisfatórios (entre .70 e .80, com excepção das escalas de Ansiedade Fóbica e do Psicoticismo, que apresentam valores de .62), quer para a generalidade dos itens, quer para os valores globais das escalas.

Para o presente estudo, foi utilizado o Índice Geral de Sintomas (IGS) que informa sobre o grau de distress psicológico global. Este índice é obtido através da soma das pontuações de todos os itens, seguida da divisão pelo número total de itens (53 itens). No estudo da validação do BSI para a realidade portuguesa, a média do IGS para população geral situou-se no $0,83(D P=0,48)$, sendo que a média para a população clínica foi de $1,43(D P=0,70)$ (Canavarro, 2007). Estes valores médios são meramente indicativos e não pode ser aferida a presença de psicopatologia através do IGS, uma vez que este índice compósito não fornece valores e pontos de corte que permitam diferenciar a população comunitária da população clínica.

Parenting Alliance Measure ([PAM], Abidin \& Konold, 1999). Os 20 itens do PAM avaliam a percepção que cada um dos pais tem da tarefa coparental com o outro progenitor do seu filho, medindo mais concretamente a comunicação entre a díade parental sobre as práticas e estilos educativos, o compromisso mútuo quanto à educação e a compreensão das dificuldades e potencialidades parentais do outro pai. $\mathrm{O}$ instrumento dirige-se aos aspectos exclusivamente relacionados com a coparentalidade, não avaliando os componentes conjugais, o que permite a sua utilização com pais divorciados. Considerando o sistema de cotação do instrumento, quanto maior for a pontuação (máximo 100), maior a aliança parental na díade. Na versão original, o PAM apresenta altos índices de validade interna, o mesmo acontecendo na versão portuguesa do instrumento $(\alpha=.92)$ (Lamela, Castro, Nunes-Costa, \& Figueiredo, 2008).

Divorce Adjustment Inventory Revised ([DAI-R], Portes, Smith, \& Brown, 2000). Este instrumento avalia o ajustamento ao divórcio. É constituído por 42 itens organizados em cinco subescalas: Conflito e Disfunção Familiares, Condições Favoráveis do Divórcio e a Capacidade de Coping da Criança, Resolução Positiva do Divórcio, Sistemas Externos de Suporto e Transição para o divórcio. No presente estudo apenas o total geral foi utilizado e não os valores das subescalas, sendo que, em questão da interpretação das pontuações obtidas, quanto maior for o score total dos participantes no instrumento, pior é a adaptação ao divórcio. Na versão norte-americana, a validade e a consistência interna é bastante razoável, com valores de alfa de Cronbach entre .65 e .84 . Este inventário foi seleccionado para a avaliação da eficácia do programa de intervenção porque colecta informações indetectáveis por outros instrumentos, uma vez que tem elevada sensibilidade para medir a percepção de riscos psicossociais na população divorciada (Dent, 2001). A versão traduzida utilizada é de Gonçalves, Lamela, Castro e Figueiredo (2006), estando em preparação a validação da escala para a população portuguesa pelos mesmos autores.

\section{Procedimentos Estatísticos}

Procedeu-se a uma análise exploratória de dados para examinar se as variáveis estavam adequadas para o uso de testes paramétricos. Depois de serem avaliados os três pressupostos subjacentes ao uso de estatística paramétrica - (a) variáveis intervalares, (b) normalidade da distribuição e (c) homogeneidade da variância - concluiu-se que estavam cumpridos os pressupostos para utilização deste tipo de testes. Foi conduzida uma análise preli-minar, através da utilização de testes de associação (Quiquadrado) e testes de diferenças (teste $t$ para amostras independentes e teste de Mann-Whitney) para garantir que o grupo experimental e o grupo de controlo não dife- 
riam estatisticamente, nem nas características demográficas, nem nas medidas de pré-intervenção.

Para analisar a eficácia do programa, foram comparados, num primeiro passo, as médias dos scores das medidas dependentes no momento pré-intervenção com as médias dos scores das mesmas medidas no momento de pós-intervenção, em ambos os grupos. Num segundo passo, foi examinado se existiam diferenças nas medidas dependentes entre o grupo experimental e o grupo de controlo no momento pós-intervenção, utilizando testes de diferenças (teste $t$ para amostras independentes, teste $t$ para amostras emparelhadas e teste de Mann-Whitney).

\section{Resultados}

\section{Comparação Pré-Teste}

Não foram identificadas diferenças significativas entre os dois grupos no género $\left(\chi^{2}(1)=0,00, p>0,05\right)$, na idade $(z=-1,27, p>0,5)$, na escolaridade $\left(\chi^{2}(1)=3,33\right.$, $p>0,05)$, na composição do agregado familiar $\left(\chi^{2}(1)=\right.$ $0,30, p>0,05)$, no número de anos que ocorreu o divórcio $(t(14)=1,78, p>0,05)$ e na regulação do poder paternal $\left(\chi^{2}(1)=1,06, p>0,05\right)$. No entanto, existe uma diferença significativa na média de idades dos filhos dos participantes $(t(14)=2,09, p<0,05)$, superior no grupo de controlo.

A comparação dos dois grupos nas medidas dependentes no momento pré-intervenção, demonstrou não existirem diferenças significativas nas médias dos resultados do Brief Symptom Inventory $(t(14)=-0,31, p>0,05)$, Parenting Alliance Measure $(t(14)=0,62, p>0,05) \mathrm{e}$ do Divorce Adjustment Inventory-Revised $(t(14)=-0,05$, $p>0,05)$ (Tabela 1).

Tabela 3

Médias e Desvios-padrão Para as Medidas BSI e PAM Por Grupo: Pré e Pós-Teste

\begin{tabular}{|c|c|c|c|c|c|c|}
\hline & \multicolumn{3}{|c|}{ Brief Symptom Inventory } & \multicolumn{3}{|c|}{ Parenting Alliance Measure } \\
\hline & $\begin{array}{c}\text { Pré-intervenção } \\
\text { Média }(D P)\end{array}$ & $\begin{array}{l}\text { Pós-intervenção } \\
\text { Média }(D P)\end{array}$ & $t(14)$ & $\begin{array}{c}\text { Pré-intervenção } \\
\text { Média }(D P)\end{array}$ & $\begin{array}{l}\text { Pós-intervenção } \\
\text { Média }(D P)\end{array}$ & $t(14)$ \\
\hline Grupo Experimental & $1,00(0,42)$ & $0,81(0,35)$ & 2,11 & $54,25(13,56)$ & $61,13(11,71)$ & $-3,83 *$ \\
\hline Grupo de Controlo & $0,93(0,50)$ & $0,91(0,48)$ & 0,60 & $58,88(16,26)$ & $58,38(16,37)$ & 0,34 \\
\hline
\end{tabular}

Nota. $* p<0,01$.

Tabela 4

Médias e Desvios-Padrão para as Medidas BSI e PAM Por Grupo: Pós-Teste

\begin{tabular}{lccc}
\hline & $\begin{array}{c}\text { Grupo Experimental } \\
(n=8) \\
\text { Média }(D P)\end{array}$ & $\begin{array}{c}\text { Grupo Controlo } \\
(n=8)\end{array}$ & \\
& Média $(D P)$ & $t(14)$ \\
\hline BSI & $0,81(0,35)$ & $0,91(0,48)$ & 0,48 \\
PAM & $61,13(11,71)$ & $58,38(16,37)$ & $-0,39$ \\
\hline
\end{tabular}

Nota. BSI = Brief Symptom Inventory (Derogatis \& Melisaratos, 1983); PAM = Parenting Alliance Measure (Abidin \& Konold, 1999).
Tabela 2

Médias e Desvios-Padrão para as Medidas Dependentes Por Grupo: Pré-Teste

\begin{tabular}{lccr}
\hline & $\begin{array}{c}\text { Grupo Experimental } \\
(n=8)\end{array}$ & $\begin{array}{c}\text { Grupo Controlo } \\
(n=8)\end{array}$ \\
& Média $(D P)$ & Média $(D P)$ & $t(14)$ \\
\hline BSI & $1,00(0,42)$ & $0,93(0,50)$ & $-0,31$ \\
PAM & $54,25(1,56)$ & $58,88(16,26)$ & 0,62 \\
DAI-R & $124(8,02)$ & $123,6(18,52)$ & $-0,05$ \\
\hline
\end{tabular}

Nota . BSI = Brief Symptom Inventory (Derogatis \& Melisaratos, 1983); PAM = Parenting Alliance Measure (Abidin \& Konold, 1999); DAI-R = Divorce Adjustment Inventory-Revised (Portes, Smith, \& Brown, 2000).

\section{Comparação Pré-Pós-Teste e Comparação Pós-Teste}

$\mathrm{Na}$ medida Brief Symptom Inventory (BSI) não foram encontradas diferenças estatisticamente significativas entre o pré e pós-intervenção, nem no grupo experimental $(t(14)=2,11, p>0,05)$, nem no grupo de controlo $(t(14)$ $=0,60, p>0,05)$ (ver Tabela 2). Também não foram encontradas diferenças significativas nas médias pósintervenção entre o grupo experimental e o grupo de controlo $(t(14)=0,48, p>0,05)$ (Tabela 3$)$.

No Parenting Alliance Measure (PAM) foi encontrada uma diferença estatisticamente significativa entre o pré e o pós-teste no grupo experimental $(t(14)=-3,83$, $p<0,01)$, mas não no grupo de controlo $(t(14)=0,34$, $p>0,05$

Não foram encontradas diferenças significativas entre os grupos experimental e de controlo nas médias dos totais no momento pós-intervenção $(t(14)=-0,37, p>.05)$. 
para pais divorciados. Na avaliação pré-teste, os grupos não diferiram nas medidas dependentes, o que significa que eram equivalentes no momento anterior à aplicação do programa.

Entre a avaliação pré e pós-teste no ajustamento ao divórcio e na aliança parental, os pais participantes no grupo experimental apresentaram melhorias que não foram observadas nos participantes do grupo de controlo no momento pós-teste, o que pode sugerir um impacto positivo da aplicação do programa na adaptação ao divórcio e no comprometimento coparental dos pais participantes. Na avaliação pós-teste foram encontradas diferenças significativas na adaptação ao divórcio, que se mostrou melhor nos participantes do grupo de intervenção, quando comparadas com os pais que não participaram na intervenção.

No entanto, o programa não contribuiu para uma diminuição estatisticamente significativa do mal-estar psicológico, medido pelo BSI, nos participantes do grupo experimental. Visto que a intervenção esteve essencialmente focada na adaptação ao divórcio e coparentalidade, não foi necessariamente eficaz na diminuição directa dos distress psicológico e dos sintomas globais de mal-estar. A título ilustrativo, os valores médios do IGS apresentados pelo grupo experimental $(1,00)$ e grupo controlo $(0,93)$, no momento pré-intervenção, colocam-nos acima do valor médio da população portuguesa $(0,83)$, o que indica que os participantes de ambos os grupos apresentavam menor bem-estar psicológico do que a maioria da população. Já no momento pós-intervenção, os valores do IGS do grupo experimental $(0,81)$ encontram-se abaixo do valor médio da população portuguesa $(0,83)$, enquanto o valor médio do IGS do grupo de controlo aumentou para um valor superior ao verificado no momento pré-intervenção, o que aponta para uma diminuição do mal-estar psicológico no grupo experimental e um aumento periférico do mal-estar psicológico no grupo de controlo. Contudo, esta análise deve ser lida com alguma precaução. Em primeiro, embora haja flutuações nos valores do IGS entre os grupos e entre os momentos de avaliação, estas diferenças não são estatisticamente significativas, não permitindo comprovar a eficácia do programa na melhoria do bem-estar psicológico. Em segundo, embora os valores apresentados pelos grupos nos dois momentos de avaliação estejam acima ou abaixo do valor médio do IGS para a população portuguesa, estes encontram-se dentro do desvio-padrão esperado.

Dessa forma, os resultados globais obtidos nesta primeira edição do PApi reforçam os princípios orientadores e os alicerces teóricos da intervenção. As vantagens deste programa foram a conjugação de características positivas de outros programas de intervenção em grupo, quer nos conteúdos temáticos, quer nos métodos de intervenção. A inovação do PApi é aliar no mesmo programa três temas fundamentais para as trajectórias desenvolvimentais adaptadas dos pais: o ajustamento ao divórcio, a coparentalidade cooperante e a binuclearidade familiar (Lamela et al., 2007b). Muitos estudos têm seleccionado estes três conceitos como essenciais na intervenção psicológica com os membros de famílias divorciadas (Arbuthnot et al., 1996; Ferrante, 2005; Gilmour, 2004; Macie, 2002; Pedro-Carroll \& Cowen, 1985).

Para além dos conteúdos, uma das vantagens deste programa são os métodos de intervenção seleccionados: um ambiente de suporte e partilha emocional, um espaço orientado para as capacidades, em que são trabalhadas e treinadas algumas competências preciosas para a mudança e introduzido, ao mesmo tempo, conhecimento psicológico aplicado a situações reais. Todas estas características foram identificadas em estudos anteriores como fundamentais, quando combinadas, para o sucesso da intervenção em grupo com pais divorciados (Bloom et al., 1982; Pereira et al., 2008).

Este estudo apresenta, contudo, algumas limitações. A primeira delas diz respeito ao reduzido número de participantes. Seguindo as orientações metodológicas propostas por Kazdin (2003), para um tipo de estudo desta natureza, seria necessário um maior número de participantes para poder avaliar com maior exactidão o impacto do PApi na melhoria do ajustamento ao divórcio e na construção de uma coparentalidade mais positiva e cooperante. Embora este estudo possa dar apenas algumas indicações sobre o efeito positivo do programa para os participantes e, consequentemente, a limitação de extrapolar seus resultados, dá a pista de que o protocolo e o método de avaliação da eficácia deste programa é funcional e que se ajusta metodologicamente.

Idealmente, será importante em futuras replicações do presente estudo, possibilitar a aleatorização dos grupos em análise, permitindo melhorar a validade do estudo da eficácia do programa. Sugere-se ainda a triangulação dos resultados quantitativos apresentados com a introdução de metodologias qualitativas de análise de dados, permitindo, por consequência, superar pequenas lacunas ao nível da explicação de alguns dos resultados encontrados, tais como a dificuldade em avaliar e descrever as mudanças nos processos desenvolvimentais de adaptação ao divórcio (e.g., percepções do self, estratégias de coping, mudança e (re)construção narrativa da adaptação à dissolução conjugal, processos de luto e de desvinculação e reorganização da vinculação), que se tornam difíceis de compreender utilizando exclusivamente métodos quantitativos de análise de dados.

Os dados apresentados dão indicações que o programa poderá ter contribuído para melhorar o ajustamento ao divórcio dos participantes, um dos objectivos cimeiros deste projecto, quer pelo efeito directo - temas abordados, treino de novas competências, o espaço experiencial providenciado nas sessões - quer pelos efeitos indirectos, como, por exemplo, através da criação de uma nova rede social de suporte que não existia até então. Richmond e Christensen (2000) sustentam que a criação, renovação 
e manutenção de novos ciclos de suporte social representam um dos pilares mais significativos para uma adaptação ao divórcio mais equilibrada e saudável. Dessa feita, não é errado inferir que o sentimento de criação de uma nova rede de apoio também poderá ter contribuído para as melhorias visíveis nos índices de ajustamento. Por consequência, deve ficar em ponderação a inserção de medidas que meçam as redes de suporte social em futuras avaliações da aplicação do programa.

Arbuthnot et al. (1996) demonstraram que os efeitos positivos do programa que desenvolveram permaneceram e melhoraram na avaliação de follow-up, um ano após à aplicação do programa de intervenção. Para estes autores, a acentuação das melhorias um ano após a participação dos pais prende-se com a natureza dos resultados a longo-prazo das técnicas de interrupção do conflito. Por outras palavras, as técnicas de interrupção de conflito que os pais aprenderam e treinaram durante o programa não têm necessariamente impacto imediato na mudança relacional da díade parental. Os pais poderão não ter conseguido identificar melhorias a curto-prazo, embora, depois de alguns meses após a intervenção, e com a existência de episódios em que as estratégias e os conhecimentos aprendidos podem ser aplicados, os pais notaram, então, uma melhoria comportamental, fazendo com que a sua percepção quanto aos efeitos positivos do programa tenha ainda melhorado. $\mathrm{O}$ efeito encontrado neste estudo vem ainda reforçar a necessidade de implementar um momento de avaliação de follow-up, que irá ocorrer em ambos os grupos um ano após a finalização da participação no programa. A introdução deste momento de avaliação de follow-up aumentaria o contributo deste estudo na investigação da eficácia de programas de intervenção com pais divorciados, uma vez que são escassos os estudos de avaliação de eficácia que introduzam o follow-up (Gilmour, 2004).

Numa outra perspectiva, esta primeira versão do $P A p i$ foi desenvolvida num grupo de pais com um nível sóciocultural médio-elevado e com um período médio de divórcio de 4 anos. Será relevante compreender se estas dimensões poderão ter tido impacto nos resultados positivos alcançados.

Consideremos, primeiramente, o tempo de divórcio e os resultados obtidos pela participação no programa. Apesar da literatura não fornece indicações claras e metódicas sobre esta questão, pensamos que os efeitos positivos de intervenções em grupo com pais divorciados parecem ter maior amplitude em participantes cujo divórcio aconteceu há mais tempo (Blaisure et al., 2006). Entre outras pesquisas, o estudo de Arbuthnot et al. (1996) é um exemplo desta asserção. Realizado com pais recém-divorciados, esta pesquisa demonstrou que os maiores efeitos positivos foram visíveis um ano após a participação no programa (i.e., um ano após o divórcio). Do ponto de vista teórico, há sustentação para este facto. Considerando o divórcio como um processo de desvinculação ao ex-cônjuge (Feeney \& Monin, 2008), os pais, ao longo do tempo, conseguem diferenciar a exrelação conjugal do passado das necessidades coparentais do presente, o que os pode colocar numa postura mais proactiva e motivadora para a mudança na qualidade da relação coparental. Assim, pensamos que os efeitos da participação no PApi por pais recém-divorciados poderiam ser de menor dimensão em comparação com os que foram apresentados por esta amostra de pais cujo divórcio ocorreu há 4 anos.

Quanto ao nível sócio-cultural dos elementos, pensamos que o PApi está desenhado para poder ser participado por pais de diferentes quadrantes sociais e culturais. Apesar da sua estruturação semi-directiva, este programa permite uma margem de adaptação dos objectivos e estratégias ao grupo intervencionado, o que possibilita aos dinamizadores apostar e aprofundar no conjunto de tarefas que melhor respondam às características dos membros do grupo. No entanto, apesar de ser possível que o estatuto sócio-cultural possa influenciar os efeitos obtidos pela participação do PApi, não encontramos investigação sistemática sobre programas similares que reúnam evidências que sustentem esta hipótese.

Neste sentido, para responder a estas suposições, estão previstas reedições do programa modificado com grupos de pais divorciados com características sócio-demográficas diversificadas entre si. Assim, num futuro próximo, o PApi será desenvolvido num grupo de pais recém-divorciados, num grupo de pais com um regime de guarda conjunta dos filhos, num grupo de pais com guarda única dos filhos menores e num grupo de pais que estiveram envolvidos em processos litigiosos de regulação das responsabilidades parentais. De igual forma, será controlada nestes grupos a variável da idade dos filhos, uma vez que torna-se relevante compreender se o desenho de intervenção do PApi está mais adequado a um período de desenvolvimento específico das crianças ou se, por outro lado, se ajusta a pais com crianças de todas as faixas etárias, tal como está conceptualmente desenhado.

Apesar do seu cariz preliminar, o presente estudo aponta algumas direcções sobre os pontos positivos do programa. A nosso ver, o estabelecimento e desenvolvimento de um sentido de coesão e de partilha experiencial entre os participantes e a transmissão de conhecimentos sobre os processos de adaptação ao divórcio funcionaram como elementos-chave para as evoluções positivas apresentadas pelos participantes no momento pós-intervenção, o que vai de encontro com as orientações de Blaisure e Geasler (2006). Por seu lado, torna-se relevante, em futuras edições do PApi, aumentar o número de actividades de treinamento de competências de gestão de conflitos interparentais. De facto, na nossa perspectiva, a inclusão de mais momentos de aprendizagem, moldagem e treinamento de competências de relacionamento familiar poderá contribuir para efeitos ainda mais positivos.

Comparando os resultados obtidos com as contribuições teóricas e empíricas apresentadas na revisão da lite- 
ratura, pode concluir-se que o PApi alcança e corresponde aos princípios conceptuais que basearam a sua planificação (Lamela et al., 2007b). O protocolo de validação deste programa incorpora e dá resposta às principais conclusões empíricas desenvolvidas nas últimas décadas por vários autores: em primeiro, este programa é conceptualmente mais rico, contribui para criar grupos de suporte adicionais, fomenta a partilha experiencial e desenvolve as competências interpessoais dos pais, empenha-se na melhoria das competências coparentais e tem como focos de intervenção directa o adulto e a díade parental e não as crianças (Stewart, 2001); e, em segundo, o programa demonstrou contribuir para um maior conhecimento dos pais sobre si próprios, sobre como desempenhar activamente as funções coparentais e qual o impacto das suas práticas no desenvolvimento dos seus filhos (Blaisure et al., 2006; Gilmour, 2004).

Neste sentido, julgamos que os quatro factores que mais contribuíram para a mudança verificada nos participantes foram o sentimento de participar num grupo em que todos os membros estavam a passar pelo mesmo processo, o sentimento de aceitação, a oportunidade de expressar sentimentos difíceis e, por último, a compreensão das dinâmicas geradas pelo divórcio nos percursos desenvolvimentais dos adultos. Estes dados preliminares estão em consonância com as conclusões de Øygard et al. (2000) que, no seu estudo de referência, identificaram igualmente estas dimensões como as mais relevantes nas mudanças positivas apresentadas pelos participantes. Dessa feita, o Pais por Inteiro demonstrou ser uma proposta válida de um programa sistemático em língua portuguesa para a intervenção psicológica com pais divorciados.

\section{Referências}

Abidin, R. R., \& Konold, T. R. (1999). Parenting Alliance Measure Professional Manual. Odessa, FL: Psychological Assessment Resources.

Ahrons, C. R. (1981). The continuing coparental relationship between divorced spouses. American Journal of Orthopsychiatry, 51, 415-428.

Arbuthnot, J., Poole, C., \& Gordon, D. (1996). Use of educational materials to modify stressful behaviours in postdivorce parenting. Journal of Divorce and Remarriage, 25, 117-137.

Arnett, J. (2001). Conceptions of the transition to adulthood: Perspectives from adolescence through midlife. Journal of Adult Development, 8, 133-143.

Blaisure, K., \& Geasler, M. (2000). The divorce education intervention model. Family Court Review, 38, 501-513.

Blaisure, K., \& Geasler, M. (2006). Educational interventions for separating and divorcing parents and their children. In M. Fine \& J. Harvey (Eds.), Handbook of divorce and relationship dissolution (pp. 575-602). Mahwah, NJ: Lawrence Erlbaum.

Bloom, B., Hodges, W., \& Caldwell, R. (1982). A preventive program for the newly separated: Initial evaluation. American Journal of Community Psychology, 10, 251-264.
Canavarro, M. C. (1999). Inventário de Sintomas Psicopatológicos - BSI. In M. R. Simões, M. Gonçalves, \& L. S. Almeida (Eds.), Testes e provas psicológicas em Portugal (Vol. II, pp. 95-109). Braga, Portugal: APPORT.

Canavarro, M. C. (2007). Inventário de Sintomas Psicopatológicos (BSI). In M. Simões, C. Machado, M. Gonçalves, \& L. Almeida (Eds), Avaliação portuguesa: Instrumentos validados para a população portuguesa (pp. 305331). Coimbra, Portugal: Quarteto.

Cookston, J., Braver, S., Griffin, W., Lusé, S., \& Miles, J. (2007). Effects of the dads for life intervention on interparental conflict and coparenting in the two years after divorce. Family Process, 46, 123-137.

Derogatis, L. R., \& Melisaratos, N. (1983). The Brief Symptom Inventory: An introductory report. Psychological Medicine, 13, 595-605.

Dent, G. (2001). The efficacy of group psychotherapy for improving children's adjustment during the divorce transition. Trinity, Canada: Trinity Western University.

Feeney, B., \& Monin, J. (2008). An attachment-theoretical perspective on divorce. In J. Cassidy \& P. Shaver (Eds.), Handbook of attachment: Theory, research, and clinical applications (pp. 934-957). New York: The Guilford Press.

Ferrante, J. (2005). Co-parenting in intact and divorced families: Its impact on young adult adjustment. Unpublished doctoral dissertation, Virginia Commonwealth University, Richmond, VA.

Frieman, B., Garon, H., \& Garon, R. (2000). Parenting seminars for divorcing parents: One year later. Journal of Divorce \& Remarriage, 33, 129-143.

Geasler, M., \& Blaisure, K. (1998). A review of divorce education program materials. Family Relations, 47, 167-175.

Gilmour, G. (2004). High-conflict separation and divorce: Options for consideration. Ottawa, Canada: Ministério da Justiça do Canadá.

Gonçalves, T., Lamela, D., Castro, C., \& Figueiredo, B. (2006). Tradução do instrumento Divorce Adjustment Inventory Revised. Manuscrito não-publicado, Universidade do Minho, Portugal.

Goodman, M., Bonds, D., Sandler, I. N., \& Braver, S. L. (2004). Parent psychoeducational programs and reducing the negative effects of interparental conflict following divorce. Family Court Review, 42, 263-279.

Instituto Nacional de Estatística. (2001). Portugueses: Menos casamentos e mais divórcios? Lisboa, Portugal: Autor.

Instituto Nacional de Estatística. (2008). Estatísticas demográficas 2007. Lisboa, Portugal: Autor.

Hetherington, E. M. (2003). Intimate pathways: Changing patterns in close personal relationships across time. Family Relations, 52, 318-331.

Hetherington, E. M., \& Kelly, J. (2002). For better or for worse: Divorce reconsidered. New York: Norton \& Co.

Kazdin, A. (2003). Research design in Clinical Psychology. Needham Heights, MA: Allyn \& Bacon.

Kramer, K., Arbuthnot, J., Gordon, D., Rousis, N., \& Hoza, J. (1998). Effects of skill-based versus information-based programs on domestic violence and parental communication. Family and Conciliation Courts Review, 36, 9-31.

Lachman, M. (2001). Handbook of midlife development. New York: Wiley.

Lamela, D. (2009). Desenvolvimento após o divórcio como estratégia de crescimento humano. Revista Brasileira de Crescimento e Desenvolvimento Humano, 19, 114-121. 
Lamela, D., Castro, M., Nunes-Costa, R., \& Figueiredo, B (2008). Validação portuguesa do Parenting Alliance Inventory: Estudo I (Amostra de Pais Divorciados). Manuscrito-não publicado, Universidade do Minho, Portugal.

Lamela, D., Gonçalves, T., Castro, M., \& Figueiredo, B. (2007a). QLSD - Questionário de Levantamento SocioDemográfico. Manuscrito não-publicado, Universidade do Minho, Portugal.

Lamela, D., Gonçalves, T., Castro, M., \& Figueiredo, B. (2007b). PApi - Manual de Aplicação. Manuscrito não-publicado, Universidade do Minho, Portugal.

Lamela, D., Gonçalves, T., Castro, M., \& Figueiredo, B. (2007c). PApi - Pais por Inteiro: Orientações Conceptuais e Metodológicas. Manuscrito não-publicado, Universidade do Minho, Portugal.

Lerner, R. (2006). Developmental science, developmental systems, and contemporary theories of human development. In W. Damon, R. Lerner (Series Eds.), \& R. Lerner (Vol. Ed.), Handbook of Child Psychology: Vol. 1. Theoretical models of human development $\left(6^{\text {th }}\right.$ ed., pp. 1-17). Hoboken, NJ: Wiley.

Maccoby, E., Depner, C., \& Mnookin, R. (1990). Coparenting in the second year after divorce. Journal of Marriage \& the Family, 52, 141-155.

Macie, K. M. (2002). Influence of co-parenting and marital status on young adult adjustment. Unpublished doctoral dissertation, Virginia Commonwealth University, Richmond, VA.

Øygard, L. (2004). Divorce support groups: What is the role of the participants' personal capital regarding adjustment to divorce? Journal of Divorce \& Remarriage, 20, 103-119.

Øygard, L., Thuen, F., \& Solvang, P. (2000). An evaluation of divorce support groups: A qualitative aproach. Journal of Divorce \& Remarriage, 32, 141-147.

Pedro-Carroll, J., \& Cowen, E. (1985). The children of divorce intervention program: An investigation of the efficacy of a school-based prevention program. Journal of Consulting and Clinical Psychology, 53, 603-611.

Pereira, A., \& Matos, M. (2008). As crianças do divórcio e a regulação litigiosa do poder paternal. In C. Machado, \& R. Abrunhosa Gonçalves (Eds.), Violência e vítimas de crime: Crianças (pp. 265-299). Coimbra, Portugal: Quarteto.

Portes, P. R., Smith, T. L., \& Brown, J. H. (2000). The Divorce Adjustment Inventory-Revised: Validation of a parental report concerning children's post-custody adjustment. Unpublished manuscript, University of Louisville, KY.

Richmond, L., \& Christensen, D. (2000). Coping strategies and postdivorce health outcomes. Journal of Divorce \& Remarriage, 34, 41-59.

Sbarra, D., \& Emery, R. (2006). In the presence of grief: The role of cognitive-emotional adaptation in contemporary divorce mediation. In M. Fine \& J. Harvey (Eds), Handbook of divorce and relationship dissolution (pp. 553-573). Mahwah, NJ: Lawrence Erlbaum.

Stone, G., McKenry, P., \& Clark, K. (1999). Father's participation in a divorce education program: A qualitative evaluation. Journal of Divorce \& Remarriage, 30, 99-113.

Stewart, R. (2001). The early identification and streaming of cases of high-conflict separation and divorce: A review. Ottawa, Canada: Ministério da Justiça do Canadá.

Sweeper, S. (2004). Adult adjustment to relationship separation. Unpublished doctoral dissertation, Griffith University, Australia.
Wang, H., \& Amato, P. (2000). Predictors of divorce adjustment: Stressors, resources, and definitions. Journal of Marriage and Family, 62, 655-668.

Zimmerman, M. (1995). Psychological empowerment: Issues and illustrations. American Journal of Community Psychology, 23, 581-599.
Recebido: 06/11/2008

$1^{a}$ revisão: $18 / 02 / 2009$

$2^{a}$ revisão: $21 / 04 / 2009$ Aceite final: $22 / 04 / 2009$ 